\title{
Diffusion-Weighted Imaging Score of the Brain Stem: A Predictor of Outcome in Acute Basilar Artery Occlusion Treated with the Solitaire FR Device
}

\author{
I. Mourand, P. Machi, E. Nogué, C. Arquizan, V. Costalat, M.-C. Picot, A. Bonafé, and D. Milhaud
}

\begin{abstract}
BACKGROUND AND PURPOSE: The prognosis for ischemic stroke due to acute basilar artery occlusion is very poor: Early recanalization remains the main factor that can improve outcomes. The baseline extent of brain stem ischemic damage can also influence outcomes. We evaluated the validity of an easy-to-use DWI score to predict clinical outcome in patients with acute basilar artery occlusion treated by mechanical thrombectomy.
\end{abstract}

MATERIALS AND METHODS: We analyzed the baseline clinical and DWI parameters of 31 patients with acute basilar artery occlusion, treated within 24 hours of symptom onset by using a Solitaire FR device. The DWI score of the brain stem was assessed with a 12-point semiquantitative score that separately considered each side of the medulla, pons, and midbrain. Clinical outcome was assessed at 180 days by using the mRS. According to receiver operating characteristic analyses, the cutoff score determined the optimal positive predictive value for outcome. The Spearman rank correlation coefficient assessed the correlation between the DWI brain stem score and baseline characteristics.

RESULTS: Successful recanalization (Thrombolysis in Cerebral Infarction 3-2b) was achieved in 23 patients (74\%). A favorable outcome ( $m R S \leq 2$ ) was observed in 11 patients $(35 \%)$. An optimal DWI brain stem score of $<3$ predicted a favorable outcome. The probability of a very poor outcome $(\mathrm{mRS} \geq 5)$ if the DWI brain stem score was $\geq 5$ reached $80 \%$ (positive predictive value) and $100 \%$ if this score was $\geq 6$. Interobserver reliability of the DWI brain stem score was excellent, with an intraclass correlation coefficient of 0.97 (95\% Cl, 0.96-0.99). The DWI brain stem score was significantly associated with baseline tetraplegia $(P=.001)$ and coma $(P=.005)$.

CONCLUSIONS: In patients with acute basilar artery occlusion treated by mechanical thrombectomy, the baseline DWI brain lesion score seems to predict clinical outcome.

ABBREVIATIONS: $\mathrm{ABAO}=$ acute basilar artery occlusion; $\mathrm{BSS}=$ brain stem score; IQR = interquartile range; $\mathrm{pc}-\mathrm{ASPECT}=$ posterior circulation Alberta Stroke Program Early $C T$ Score; PPV = positive predictive value; $R O C$ = receiver operating characteristic

A cute basilar artery occlusion $(\mathrm{ABAO})$ is an uncommon cause of stroke, accounting for only $6 \%-10 \%$ of large-vessel strokes. ${ }^{1}$ Its prognosis is very poor, with a mortality rate of up to $85 \%{ }^{2}$ and severe residual deficits in $>65 \%$ of survivors. ${ }^{3}$

Early recanalization remains the main important factor that can improve outcome. ${ }^{4-6}$ However, even though the best recanalization rate is achieved by using a newer stent retriever, such as a

Received June 21, 2013; accepted after revision October 14.

From the Departments of Neurology (I.M., C.A., D.M.) and Neuroradiology (P.M., V.C., A.B.), University Hospital Center of Montpellier, Gui de Chauliac Hospital, Montpellier, France; and Unit of Clinical Research and Epidemiology (E.N., M.-C.P.), Department of Medical Information, University Hospital Center of Montpellier, Lapeyronie Hospital, Montpellier, France.

A. Bonafé and D. Milhaud contributed equally to this work.

Please address correspondence to I. Mourand, MD, Neurology Department, CHRU, Gui de Chauliac Hospital, 80 Avenue Augustin Fliche, 34295 Montpellier Cedex 5, France; e-mail: i-mourand@chu-montpellier.fr

http://dx.doi.org/10.3174/ajnr.A3870
Solitaire FR Device (Covidien, Irvine, California) (up to $100 \%),{ }^{7-10}$ stent retriever impact on functional outcome is not as clear-cut as expected and has not been documented in a randomized trial.

The extent of baseline brain stem ischemic damage can also influence outcome. DWI and perfusion-weighted imaging have demonstrated prognostic value in acute anterior ischemic stroke. $^{11,12}$ In previous studies on vertebrobasilar ischemic stroke, the acute global DWI lesion volume did not correlate with the baseline NIHSS score. ${ }^{13,14}$ More recently, investigators have studied the functional outcome and extent of lesions in patients with a posterior circulation infarction by diffusion-weighted MR imaging. ${ }^{15-17}$ Because of the high attenuation of the anatomic architecture in the brain stem, lesion location rather than lesion volume can be critical in determining functional outcome after a posterior circulation infarction, especially when the brain stem is involved. $^{18}$ 
The aim of our study was to test the reproducibility and validity of a novel baseline and easy-to-use prethrombectomy DWI brain stem score (BSS) in 31 consecutive patients with proved $\mathrm{ABAO}$ and treated within 24 hours of symptom onset, by using mechanical thrombectomy. This score may predict functional outcomes and help determine the indications for an optimal recanalization approach.

\section{MATERIALS AND METHODS}

From November 2009 to May 2011, consecutive patients who underwent MR imaging before a mechanical thrombectomy, with or without IV thrombolysis for ABAO, in our academic center were included in this prospective monocenter study.

Inclusion criteria were the following: 1) acute stroke in the posterior circulation within 24 hours of symptom onset, 2) MR imaging that included DWI before treatment, 3) acute basilar ischemia assessed on MR imaging matching clinical symptoms, and 4) ABAO confirmed by intra-arterial DSA and treated by using mechanical thrombectomy. Patients were excluded under the following conditions: 1) ineligibility for MR imaging; 2) pregnancy; 3 ) younger than 18 years of age; 4 ) cerebral imaging revealing an intracranial hemorrhage, a tumor, or a subacute infarct; and 5) life expectancy of $<3$ months.

The study was approved by the local ethics committee; informed consent was obtained from all patients or their relatives before treatment. The NIHSS score and the Glasgow Coma Scale were assessed by a stroke neurologist at admission and just before thrombectomy. An NIHSS score of 38 was assigned to an unconscious/comatose patient. Demographic characteristics, stroke presentation and severity, time of symptom onset, and imaging data were prospectively collected.

\section{Imaging Protocol}

MR imaging was performed as soon as possible after admission, by using a 1.5T scanner (Intera, Release 10; Philips Healthcare, Best, the Netherlands) with 33-mT/m hypergradients. MR imaging was available during the study period 24 hours per day, including outside workings hours. The MR imaging protocols, acquired in an axial section that covered the whole brain, included the following: 1) a $\mathrm{T} 2{ }^{\star}$-weighted gradient-echo sequence (to screen for intracranial hemorrhage); 2$)$ a DWI sequence ( $b=0$, $b=500$, and $1000 \mathrm{~s} / \mathrm{mm}^{2}$ to identify the necrotic core) with apparent diffusion coefficient map reconstruction; 3) fluid-attenuated inversion recovery (to corroborate information about the time from symptom onset); and 4) the time-of-flight sequence that focused on the circle of Willis (to screen for large-vessel occlusion). Collateral flow of the distal basilar artery was evaluated by CTA or MRA according to the classification of Archer and Horenstein. ${ }^{19}$

\section{Image Analyses: DWI Brain Stem Score}

A 12-point semiquantitative BSS evaluated the extent of the DWI brain stem lesions by using prethrombectomy MR imaging. Our new score was based on direct visual interpretation of the DWI. To assess the score, we analyzed each brain stem level (medulla, pons, and midbrain) separately and considered the right side and the left side separately. One point was given for each unilateral high-intensity lesion at the brain stem level (medulla, pons, and midbrain) that occupied less than one-half of the area; 2 points were given if the lesion occupied more than one-half of the area. To obtain higher interobserver reproducibility, we chose the slide on which the lesion was more evident and more extended. The brain stem DWI lesion score ranged from 0 to 12. A DWI BSS of zero indicated the absence of visible brain stem ischemia, whereas a score of 12 indicated ischemic lesions in all the brain stem territories. Two examples of scoring are given in Figs 1 and 2 .

To assess interobserver reproducibility, a neuroradiologist (P.M.) and 2 neurologists (D.M., I.M.), blinded to the clinical outcomes, each independently read each patient's diffusionweighted MR images and assessed the DWI BSS. Moreover, a neuroradiologist (P.M.) and a neurologist (I.M.) assessed the posterior circulation Alberta Stroke Program Early CT Score (pcASPECTS) on DWI according to the method described by Tei et $\mathrm{al}^{17}$ and the DWI score proposed by Renard et al. ${ }^{15}$

\section{Therapeutic Regimens}

Selective diagnoses and therapeutic DSA were performed in all cases with the patient under general anesthesia. Treatment of ABAO involved either a mechanical thrombectomy after IV thrombolysis (as a bridging therapy $[n=19]$ at a full dose 0.9 $\mathrm{mg} / \mathrm{kg}$, with a loading dose of $10 \%$ ) or a mechanical thrombectomy alone $(n=12)$ in patients with a contraindication for IV thrombolysis according to the European Cooperative Acute Stroke Study (ECASS) III. ${ }^{20}$ Mechanical thrombectomy was performed by using a Solitaire FR device, which was certified through the Conformité Européenne for thrombectomy. It is a laser-cut, self-expanding, and fully retrievable split-design nitinol device. The TICI scale was used to assess recanalization of the basilar artery. Antiplatelet drugs were started 24 hours after IV thrombolysis and after CT or MR imaging.

\section{Outcome Measures}

Successful recanalization was defined as a TICI grade of $2 b$ or 3 . Symptomatic intracranial hemorrhage was defined as a documented hemorrhage associated with a decline of $\geq 4$ points in the NIHSS score. Device-related complications included vessel perforation, arterial dissection, or distal embolization of a previously uninvolved region. Clinical follow-up was assessed at 30, 90, and 180 days. Because outcome tends to be better for patients with ABAO after a longer follow-up, clinical outcome was assessed by using the $\geq 180$-day NIHSS score, the mRS, the Barthel Index, and mortality rates. A favorable outcome was defined as a $\geq 180$-day $\mathrm{mRS}$ score of $0-2$. A very poor outcome, including death and total dependency, was defined as a mRS score of 5-6.

\section{Statistical Analyses}

The intraclass correlation coefficient was used to assess the intraobserver reproducibility of the DWI BSS. Statistical associations between DWI BSS and baseline characteristics were evaluated by using the Spearman rank correlation coefficient and the Fisher exact test. For the 3 scores, receiver operating characteristic analysis (ROC) curves were drawn by plotting sensitivity against the 1-specificity of each possible cutoff point of the score. The area under the ROC curve was calculated along with its 95\% confi- 

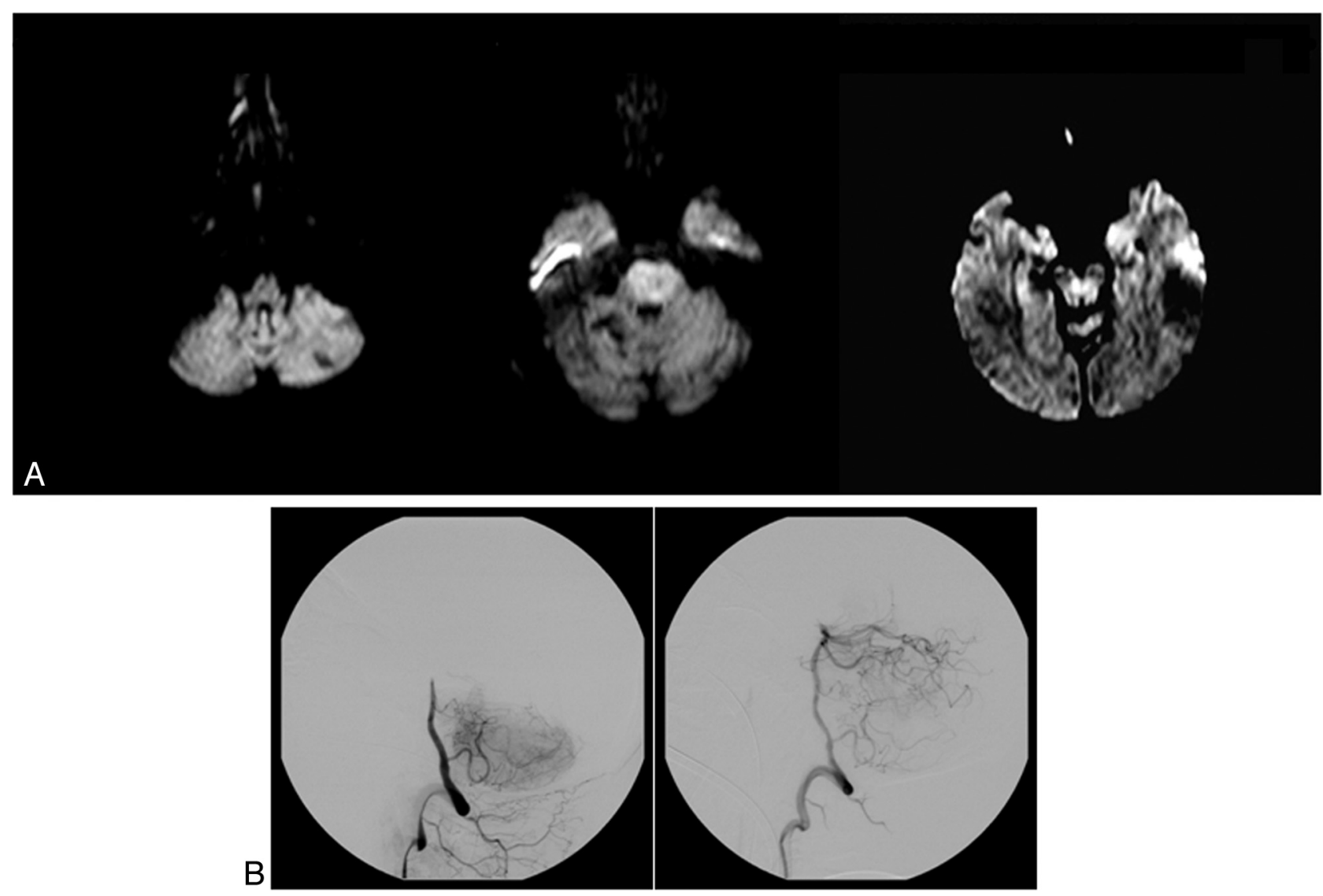

FIG 1. Prethrombectomy DWI $(A)$ of a 54-year-old woman with tetraplegia in a coma. The DWI shows extensive bilateral lesions in the pons and limited bilateral lesions in the midbrain (brain stem score, $2+2+1+1=6$ ). DSA shows a midbasilar occlusion $(B)$ successfully recanalized after IV thrombolysis and a thrombectomy ( $\mathrm{TICl} 3$ ). Time from symptom onset to recanalization was 283 minutes (4 hours 43 minutes). The outcome was unfavorable by day 180 (mRS 5).

dence interval and was compared among the 3 scores by using the method of DeLong et al. ${ }^{21}$ The optimal cutoff point was determined to increase to a maximum to the Youden Index (the difference between the true-positive and the false-positive rates), and the positive predictive value (PPV), to predict a favorable outcome ( $\mathrm{mRS} 0-2$ ) and a very poor outcome (mRS 5-6). Potential independent predictors were tested in a multivariate logistic regression including the DWI BSS. The OR and its 95\% CI were reported. The statistically significance threshold was set at $5 \%$. Statistical analyses were conducted by using SAS software, Version 9.1 (SAS Institute, Cary, North Carolina).

\section{RESULTS}

Thirty-one consecutive patients with ABAO met the inclusion criteria of our study. Their prethrombectomy clinical status was severe, with a median NIHSS score of 38 (interquartile range $[\mathrm{IQR}]=9-38)$.

Baseline characteristics, recanalization rates, procedural complications, and clinical outcomes are summarized in $\mathrm{Ta}$ bles 1 and 2 .

The median time interval from symptom onset to MR imaging was 334 minutes (IQR $=160-480$ minutes). Patients had a lesion detected by DWI in the following locations: $1(3.2 \%)$ in the medulla, $27(8.7 \%)$ in the pons, $14(45.2 \%)$ in the midbrain, 8 $(25.8 \%)$ in the temporo-occipital area, $7(22.6 \%)$ in the thalamus, and $17(54.8 \%)$ in the cerebellum. All patients, except 1, presented with at least 1 lesion of the brain stem, including 16 patients with a bilateral lesion of the pons and 4 patients with a bilateral lesion of the midbrain. Twenty-one patients $(67.7 \%)$ had multiple lesions.

The median DWI BSS was 4 (IQR = 2-4; range, $0-8)$. Twentyone patients had a score of $\geq 3(68 \%)$; no patient had a score $>8$. The mean time to assess the DWI BSS was 136 seconds (range, 16-300 seconds). On the DWI, the median pc-ASPECTS was 6 $(\mathrm{IQR}=4-8$; range $=2-10)$ and the median Renard et al ${ }^{15}$ score was $3(\mathrm{IQR}=2-4.5$; range $=0-6)$.

All patients showed complete occlusion of the basilar artery (TICI 0). Only 1 patient presented with a distal vertebral artery occlusion and a proximal basilar occlusion. For patients with a bridging therapy, the median door-to-needle time was $97.5 \mathrm{~min}$ utes ( $\mathrm{IQR}=89-150$ minutes) and the median time from the start of intravenous thrombolysis to groin puncture was 40 minutes $(\mathrm{IQR}=20-103)$. The mean time from symptom onset to recanalization was $512 \pm 258$ minutes.

Among the 10 embolic infarcts observed in a new territory, 2 patients had severe neurologic deterioration or death and 5 patients experienced mild-to-moderate clinical sequelae (gait ataxia, abnormal visual field). One patient among the 5 with symptomatic intracranial hemorrhages died from a large symp- 

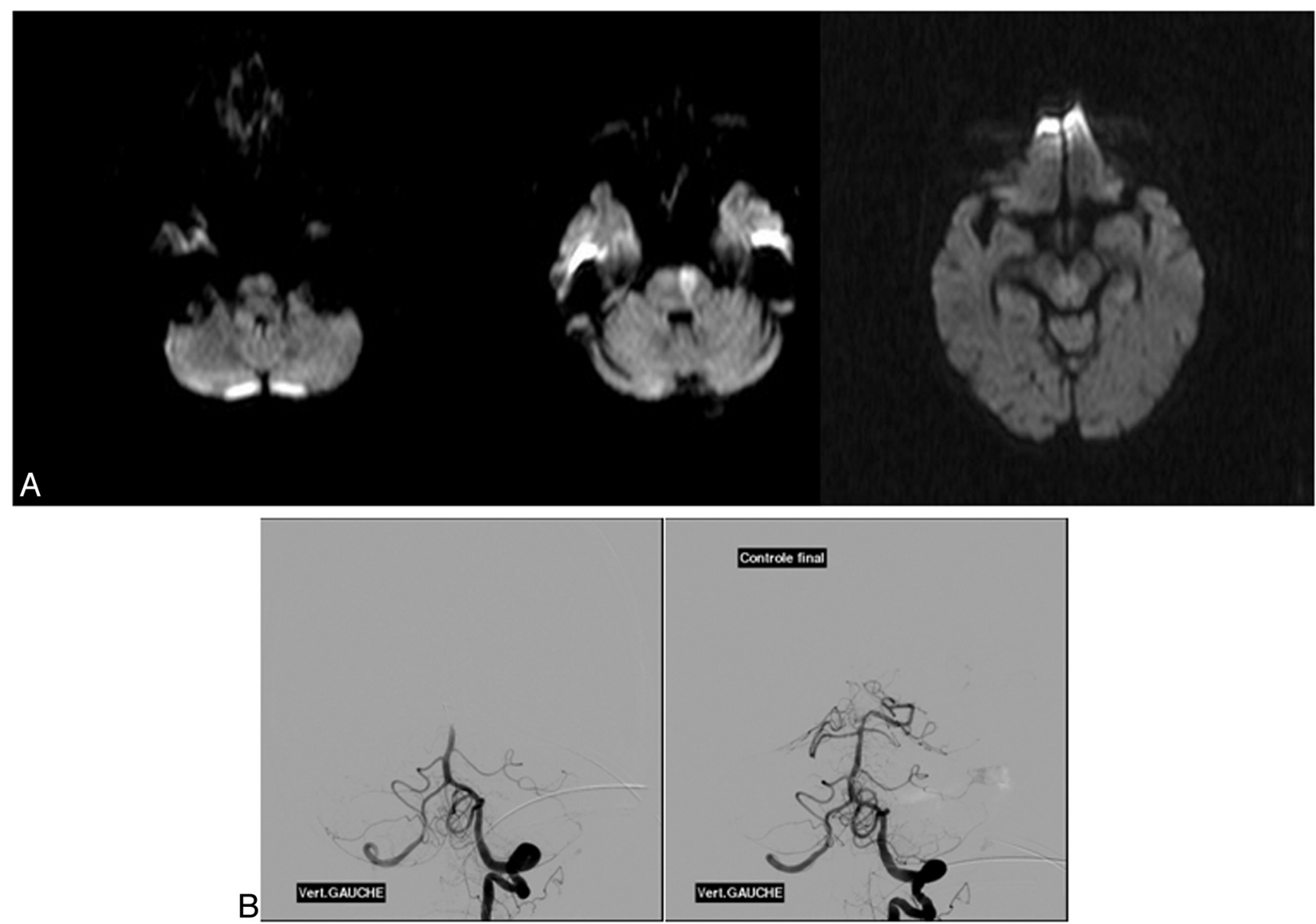

FIG 2. An 88-year-old woman who had right hemiparesis, vertigo, dysarthria, and vomiting. Pretreatment DWI ( $A$ ) shows an extensive lesion in the left pons (brain stem score, 2) with a distal basilar clot on DSA (B) recanalized after IV thrombolysis and a thrombectomy (TICI 3). The time from symptom onset to recanalization was 336 minutes (4 hours 63 minutes). At day 180, the outcome was favorable (mRS 0).

Table 1: Baseline clinical and radiologic characteristics of 31 patients

\begin{tabular}{|c|c|c|c|}
\hline Clinical Characteristics & & \multicolumn{2}{|c|}{ Radiologic Characteristics (No.) (\%) } \\
\hline Mean age (yr) & $61 \pm 17$ & Site of basilar artery occlusion & \\
\hline Men (No.) (\%) & $15(48)$ & Proximal & $4(13)$ \\
\hline Median NIHSS ${ }^{\mathrm{a}}$ (IQR) & $38(9-38)$ & Mid & $8(26 \%)$ \\
\hline Median Glasgow Coma Scale ${ }^{a}$ (IQR) & $7(4-14)$ & Distal & $19(61 \%)$ \\
\hline Tetraplegia (No.) (\%) & $11(35.5)$ & Presence of collateral flow & $19(61)$ \\
\hline Coma (GCS $\leq 8)$ (No.) (\%) & $19(61)$ & & \\
\hline Cardioembolic infarct (No.) (\%) & $16(52)$ & & \\
\hline
\end{tabular}

Note:-GCS indicates Glasgow Coma Scale.

${ }^{\text {a }}$ Prethrombectomy.

Table 2: Outcome characteristics (recanalization rate, procedural complications, and clinical outcome) of 31 patients

\begin{tabular}{lclc}
\hline Recanalization rate (TICI 3-2b) & $23(74 \%)$ & $\begin{array}{c}\text { Clinical outcome } \geq 180 \text { days } \\
\text { Median NIHSS (IQR) }\end{array}$ & $3(2-6)$ \\
Procedural complications & & Median Barthel Index (IQR) & $100(45-100)$ \\
New embolic infarcts & $10(32 \%)$ & Median mRS (IQR) & $4(2-6)$ \\
Intracranial hemorrhage symptomatic & $5(16 \%)$ & mRS 0-2 & $11(35 \%)$ \\
Other & & mRS 3-5 & $10(32 \%)$ \\
\hline
\end{tabular}

tomatic posterior fossa hematoma. Only 1 device-related complication occurred, a postprocedural vertebral dissection with posterior inferior cerebellar artery infarction.

\section{Predictive Factors for Clinical Outcome}

Univariate analysis failed to demonstrate any statistical significance among coma, tetraplegia, and the clinical outcome. Only $18 \%$ of patients presenting with tetraplegia had a favorable outcome $(\mathrm{mRS} \leq 2)$, while $82 \%$ of patients presenting without tetraplegia had a favorable outcome $(\mathrm{OR}=4.05[0.69-23.95] ; P=.12)$. Furthermore, $68 \%$ of patients presenting with coma had an unfavorable outcome, while $32 \%$ of patients presenting without coma had unfavorable outcome $(\mathrm{OR}=1.80[0.39-8.35], P=.45)$. 
Table 3: Statistical measures of the cutoff performance for the 3 scores

\begin{tabular}{|c|c|c|c|c|c|c|}
\hline Scores & $\operatorname{AUC}(95 \% \mathrm{Cl})$ & Cutoff & Sensitivity & Specificity & PPV & NPV \\
\hline \multicolumn{7}{|c|}{ Favorable outcome (mRS $\leq 2$ ) } \\
\hline DWI BSS & $0.76(0.58-0.95$ & $<3$ & $64 \%$ & $85 \%$ & $70 \%$ & $81 \%$ \\
\hline Pc-ASPECTS & $0.63(0.41-0.84)$ & $\geq 7$ & $55 \%$ & $65 \%$ & $46 \%$ & $72 \%$ \\
\hline Renard et $\mathrm{al}^{15}$ & $0.73(0.53-0.92$ & $<3.5$ & $82 \%$ & $65 \%$ & $56 \%$ & $87 \%$ \\
\hline \multicolumn{7}{|c|}{ Very poor outcome (mRS $\geq 5$ ) } \\
\hline \multirow[t]{5}{*}{ DWI BSS } & $0.80(0.63-0.96)$ & $\geq 2$ & $100 \%$ & $41 \%$ & $58 \%$ & $100 \%$ \\
\hline & & $\geq 3$ & $93 \%$ & $53 \%$ & $62 \%$ & $90 \%$ \\
\hline & & $\geq 4$ & $79 \%$ & $65 \%$ & $65 \%$ & $79 \%$ \\
\hline & & $\geq 5$ & $29 \%$ & $94 \%$ & $80 \%$ & $62 \%$ \\
\hline & & $\geq 6$ & $21 \%$ & $100 \%$ & $100 \%$ & $61 \%$ \\
\hline
\end{tabular}

Note:-NPV indicates negative predictive value.

Table 4: Functional outcome according to the categorized diffusion-weighted imaging brain stem score

\begin{tabular}{lrlccc}
\hline DWI BSS & No. & mRS 0-2 & mRS 3-4 & mRS 5-6 & P Value $^{\text {a }}$ \\
\hline $0-2$ & 10 & $7(70 \%)$ & $2(20 \%)$ & $1(10 \%)$ & .05 \\
$3-5$ & 18 & $4(22 \%)$ & $6(33 \%)$ & $8(44 \%)$ & \\
$6-12$ & 3 & $0(0 \%)$ & $0(0 \%)$ & $3(100 \%)$ & \\
Total & 31 & $11(35 \%)$ & $6(19 \%)$ & $14(45 \%)$ & \\
\hline
\end{tabular}

${ }^{\text {a }}$ Fisher $P$ value.

Patients with successful recanalization showed a nonsignificant trend toward a better outcome $(P=.20)$. Moreover, the mean time from symptom onset to recanalization tended to be lower in our patients with a poor outcome compared with patients with a favorable outcome $(P=.03)$. New embolic infarcts $(P=1)$ or symptomatic intracranial hemorrhage $(P=.13)$ did not reach significance to predict clinical outcome.

\section{Correlation between Scores and Clinical Variables}

The DWI BSS was moderately correlated with the score of Renard et $\mathrm{al}^{15}(r=0.63)$ and was weakly correlated with the pc-ASPECTS $(r=-0.38)$, whereas a strong correlation appeared between the pc-ASPECTS and the score of Renard et al $(r=-0.88)$.

The DWI BSS was significantly higher in the presence of tetraplegia $(P=.001)$ or coma $(P=.005)$ at admission. No relation was found between this score and age, the baseline NIHSS and Glasgow Coma Scale scores, time interval from symptom onset to MR imaging, presence of collateral flow, use of IV thrombolysis, the recanalization rate, new embolic infarct, or symptomatic intracranial hemorrhage.

\section{Predictors}

Brain stem DWI lesion measurements demonstrated a good reproducibility with an intraclass correlation coefficient of 0.98 (95\% CI, 0.96-0.99). The area under the curve, which approximated the chance that the DWI BSS correctly predicted a favorable outcome (mRS $<2$ ), was 0.76 (95\% CI, 0.60-0.93). It was better but not significantly different $(P=.25)$ from the score of Renard et $\mathrm{al}^{15}$ (area under the curve $=0.73$ ) and the pc-ASPECTS (area under the curve $=0.63$ ). According to the ROC, the optimal cutoff score to predict a favorable outcome was 3 for DWI BSS, 3.5 for the score of Renard et al, and 7 for pc-ASPECTS. Associated performances are presented in Table 3 . In fact, the probability of having a favorable outcome reached $70 \%$ if the DWI BSS was $<3$ (PPV). The functional outcomes, according to the DWI BSS, are presented in Table 4.

In a univariate model, a DWI BSS of $<3$ and a lesion score of
Table 5: Predictors of favorable outcome ( $m R S \leq 2$ ) analyzed by univariate regression logistic

\begin{tabular}{lccc}
\hline & OR & 95\% Cl & $P$ Value \\
\hline Age $($ yr) & 0.96 & $0.91-1.01$ & .08 \\
DWI BSS $<3$ & 9.92 & $1.75-56.30$ & .01 \\
pc-ASPECTS $\geq 7$ & 2.23 & $0.50-10.00$ & .30 \\
Renard et $\mathrm{al}^{15}<3.5$ & 8.36 & $1.40-49.88$ & .02 \\
\hline
\end{tabular}

Renard et $\mathrm{al}^{15}$ of $<3.5$ were predictors of a good outcome (mRS $<$ 2 ), whereas a pc-ASPECTS of $\geq 7$ was not statistically relevant (Table 5). In the multivariate analysis, age, the baseline Glasgow Coma Scale score, DWI BSS of $<3$, Renard et al ${ }^{15}$ score $<3.5$, and IV thrombolysis were tested. Only a DWI BSS of $<3$ and age were considered predictive of favorable outcome $\left(\mathrm{OR}_{\mathrm{DWI}<3}=\right.$ $18.4\left[95 \%\right.$ CI, 2.1-162.4] and $\mathrm{OR}_{\mathrm{Age}}=0.93$ [95\% CI, 0.97-0.10]).

Last, the probability of having a very poor outcome ( $\mathrm{mRS}>5$ ) if the DWI BSS was $>5$ reached $80 \%$ (PPV) and $100 \%$ if this score was $>6$ (Table 3 ). With these properties associated with an area under the curve of 0.80 , the DWI BSS appeared more relevant than the score of Renard et $\mathrm{al}^{15}$ (area under the curve $=0.71$ ) and the pc-ASPECTS (area under the curve $=0.59$ ) for predicting very poor outcome.

\section{DISCUSSION}

This study reports the largest baseline DWI data available on $\mathrm{ABAO}$ and shows that our new BSS could predict clinical outcome without needing additional time-consuming postprocessing.

In posterior circulation stroke, previous studies have failed to detect a correlation between lesion volume detected by DWI and the neurologic scale at onset ${ }^{13}$ or functional outcome. ${ }^{18}$ More recently, authors ${ }^{15-17}$ have studied patients with a posterior circulation stroke by using various neuroradiologic scales of DWI. In 2010, Tei et $\mathrm{al}^{17}$ evaluated the usefulness of the pc-ASPECTS on DWI to predict functional outcome in 132 patients with a posterior circulation infarction. They found that the pc-ASPECTS was an independent predictor of functional outcome $(P<.001)$. According to the ROC, the optimal cutoff score that predicted a favorable outcome was a pc-ASPECTS of $\geq 7$ and an NIHSS score of $\leq 5$. Renard et $\mathrm{al}^{15}$ reported 16 patients with $\mathrm{ABAO}$ who received intra-arterial thrombolysis. Using a 10-point semiquantitative DWI score, they found that in univariate analyses, all patients with a lesion score of $>3$ had a worse outcome or died $(P=$ .026). Cho et $\mathrm{al}^{16}$ analyzed 29 patients treated with endovascular procedures for $\mathrm{ABAO}$ and found that the brain stem score was the only independent baseline predictor of clinical outcome. In contrast to previous studies ${ }^{13,14}$ on vertebrobasilar ischemic stroke, 
they found a significant association between the DWI brain stem lesion score and the baseline NIHSS score, as well as between the presence and length of a coma before treatment.

The results of Cho et $\mathrm{al}^{16}$ support the use of sectorial scoring of DWI lesions in ABAO rather than global volume assessment. However, these previous reports have some limitations. The Renard et $\mathrm{al}^{15}$ score and the pc-ASPECTS ${ }^{17}$ included more items than our score (ie, they included an infarction in the cerebellum, thalamus, or temporo-occipital lobe). These lesions are less involved in functional prognoses than those in the brain stem. Effectively, small strategic brain stem infarcts can cause devastating clinical syndromes, whereas large cerebellar infarcts may cause minimal symptoms and attenuate the strength of the relationship between lesion volume and clinical functional status. ${ }^{14}$ For instance, with the pc-ASPECTS, a patient with a large bilateral pontine infarction has the same lesion score (of 2) as a patient with a small bilateral cerebellar infarction, but the former condition is likely to have a worse prognosis. ${ }^{15}$ Moreover, patients lose the same number of points if they present with an extensive lesion of the structure as well as a punctiform infarct. To improve correlations with mRS, which is essentially based on motor function, Renard et $\mathrm{al}^{15}$ gave a higher score in lesion structures that contain a pyramidal tract. These findings suggest that the main benefit of DWI analysis remains the brain stem, essentially because involvement of the brain stem, in particular the pons and midbrain, is critical to functional outcome.

The use of a brain stem score to identify a predictor of clinical outcome had been tested by Cho et al. ${ }^{16}$ Their score is based on arterial territory segmentation of the medulla, pons, and midbrain, as described by Tatu et al. ${ }^{22}$ However, they did not determine the optimal cutoff score, according to ROC, to predict outcome. Moreover, MR imaging analysis of the exact volume of a lesion in a brain stem score appears difficult to assess routinely as a standard score in an acute phase, especially by using DWI sequences, which have poor spatial resolution.

In our population of patients with ABAO, a pc-ASPECTS of $\geq 7$ was not statistically associated with the favorable clinical outcome. However, our population did have specific differences. In the study of Tei et al, ${ }^{17}$ all the arteries within the posterior territory were involved and there was no exclusive ABAO, acute arterial occlusion was not proved, and no patients received acute revascularization therapy. In addition, their patients had less severe symptoms, and the pc-ASPECTS was evaluated within 12-36 hours after stroke onset. ${ }^{17}$ This time window is very long compared with that in current thrombolytic therapies.

The use of DWI BSS may help to identify patients who could benefit from recanalization therapy. Our aim was to simplify the decision-making algorithm, focusing on the DWI BSS, evaluated only at the level of the brain stem, because it seems to be the most critical area for functional outcome. DWI BSS is a rapid and easyto-use score, with an excellent interobserver reliability. Furthermore, in comparison with previous scores published in the literature, the DWI BSS appeared to relate better to good and poor outcomes, respectively. Looking at the results of our study, we can state that patients with ABAO and a DWI BSS of $<3$ are good candidates for mechanical revascularization. Conversely, basilar artery recanalization of patients with DWI BSS of $\geq 6$ is not re- lated to good clinical outcome. Hence, patients in this group should not be considered for mechanical treatment.

The size of our sample is relatively small. Further studies are required in a larger $\mathrm{ABAO}$ population to evaluate whether the DWI BSS is a reliable predictor for clinical outcome or to evaluate other MR imaging parameters to identify patients who can benefit from recanalization therapy.

\section{CONCLUSIONS}

This report shows that a DWI BSS is useful in predicting outcomes in ABAO treated by thrombectomy and may simplify the decision-making algorithm. However, further evaluation is needed before it can be used as a routine treatment strategy.

Disclosures: Alain Bonafé-UNRELATED: Consultancy: Covidien, Comments: consultant for ev3 Inc, Grants/Grants Pending: Covidien.* Vincent CostalatUNRELATED: Consultancy: Balt, Codman, Stryker, Payment for Lectures (including service on Speakers Bureaus): Stryker, Balt, Payment for Development of Educational Presentations: Covidien. *Money paid to the institution.

\section{REFERENCES}

1. Mortimer AM, Bradley M, Renowden SA. Endovascular therapy for acute basilar artery occlusion: a review of the literature. J Neurointervent Surg 2012;4:266-73

2. Mattle HP, Arnold M, Lindsberg PJ, et al. Basilar artery occlusion. Lancet Neurol 2011;10:1002-14

3. Schonewille WJ, Algra A, Serena J, et al. Outcome in patients with basilar artery occlusion treated conventionally. J Neurol Neurosurg Psychiatry 2005;76:1238-41

4. Eckert B, Kucinski T, Pfeiffer G, et al. Endovascular therapy of acute vertebrobasilar occlusion: early treatment onset as the most important factor. Cerebrovasc Dis 2002;14:42-50

5. Arnold M, Nedeltchev K, Schroth G, et al. Clinical and radiological predictors of recanalisation and outcome of 40 patients with acute basilar artery occlusion treated with intra-arterial thrombolysis. J Neurol Neurosurg Psychiatry 2004;75:857-62

6. Lutsep HL, Rymer MM, Nesbit GM. Vertebrobasilar revascularization rates and outcomes in the MERCI and multi-MERCI trials. $J$ Stroke Cerebrovasc Dis 2008;17:55-57

7. Andersson T, Kuntze Soderqvist A, Soderman M, et al. Mechanical thrombectomy as the primary treatment for acute basilar artery occlusion: experience from 5 years of practice. J Neurointerv Surg 2013;5:221-25

8. Espinosa de Rueda G, Parrilla J, Zamarro B, et al. Treatment of acute vertebrobasilar occlusion using thrombectomy with stent retrievers: initial experience with 18 patients. AJNR Am J Neuroradiol 2013;34:1044-48

9. Mordasini P, Brekenfeld C, Byrne JV, et al. Technical feasibility and application of mechanical thrombectomy with the Solitaire FR revascularization device in acute basilar artery occlusion. AJNR Am J Neuroradiol 2013;34:159-63

10. Roth C, Mielke A, Siekmann R, et al. First experiences with a new device for mechanical thrombectomy in acute basilar artery occlusion. Cerebrovasc Dis 2011;32:28-34

11. Albers GW, Thijs VN, Wechsler L, et al, for the DEFUSE investigators. Magnetic resonance imaging profiles predict clinical response to early reperfusion: the diffusion and perfusion imaging evaluation for understanding stroke evolution (DEFUSE) study. Ann Neurol 2006;60:508-17

12. Nezu T, Koga M, Kimura K, et al. Pretreatment ASPECTS on DWI predicts 3-month outcome following rt-PA: SAMURAI rt-PA registry. Neurology 2010;75:555-61

13. Linfante I, Llinas RH, Schlaug G, et al. Diffusion-weighted imaging and National Institutes of Health Stroke Scale in the acute phase of posterior-circulation stroke. Arch Neurol 2001;58:621-28

14. Ostrem JL, Saver JL, Alger JR, et al. Acute basilar artery occlusion: diffusion- 
perfusion MRI characterization of tissue salvage in patients receiving intra-arterial stroke therapies. Stroke 2004;35:e30-e34

15. Renard D, Landragin N, Robinson A, et al. MRI-based score for acute basilar artery thrombosis. Cerebrovasc Dis 2008;25:511-16

16. Cho TH, Nighoghossian N, Tahon F, et al. Brain stem diffusionweighted imaging lesion score: a potential marker of outcome in acute basilar artery occlusion. AJNR Am J Neuroradiol 2009; 30:194-98

17. Tei H, Uchiyama S, Usui T, et al. Posterior circulation ASPECTS on diffusion-weighted MRI can be powerful marker for predicting functional outcome. J Neurol 2010;257:767-73

18. Engelter ST, Wetzel SG, Radue EW, et al. The clinical significance of diffusion-weighted MR imaging in infratentorial strokes. Neurology 2004;62:574-80

19. Archer CR, Horenstein S. Basilar artery occlusion: clinical and radiological correlation. Stroke 1977;8:383-90

20. Hacke W, Markku K, Bluhmki E, et al, for the ECASS Investigators. Thrombolysis with alteplase 3 to 4.5 hours after acute ischemic stroke. N Engl J Med 2008;359:1317-29

21. DeLong ER, DeLong DM, Clarke-Pearson DL. Comparing the areas under two or more correlated receiver operating characteristic curves: a nonparametric approach. Biometrics 1988;44:837-45

22. Tatu L, Moulin T, Bougousslavsky J, et al. Arterial territories of human brain: brainstem and cerebellum. Neurology 1996;47:1125-35 